PROCEEDINGS OF THE

AMERICAN MATHEMATICAL SOCIETY

Volume 126, Number 6, June 1998, Pages 1857-1866

S $0002-9939(98) 04184-7$

\title{
CONNECTEDNESS OF THE BASIN OF ATTRACTION FOR RATIONAL MAPS
}

\author{
KRZYSZTOF BARAŃSKI
}

(Communicated by Mary Rees)

\begin{abstract}
We prove some results concerning degree of a rational map on the immediate basin $B(s)$ of an attracting fixed point $s$. In particular, if $B(s)$ contains all but two critical points or values counted with multiplicity, then the entire basin of attraction is connected. For any number $k \geq 3$ we give examples of rational maps with disconnected basin of an attracting fixed point such that there are exactly $k$ critical points outside the immediate basin of attraction.
\end{abstract}

\section{INTRODUCTION AND RESULTS}

This paper deals with the problem of estimating the degree of a rational map on the immediate basin of an attracting fixed point. We prove the following:

Theorem 1.1. Let $f: \widehat{\mathbb{C}} \rightarrow \widehat{\mathbb{C}}$ be a rational map such that $f$ has an attracting fixed point s. Let $E$ be an open connected set contained in the immediate basin of attraction $B(s)$ such that $s \in E$ and $\partial E$ consists of a finite number of disjoint Jordan curves. Suppose there exists a component $F$ of $f^{-1}(E)$ containing $\mathrm{cl} E$, and $\operatorname{deg} f=1$ on every component of $\partial F$. Then either $B(s)$ contains at least one point from $f^{-1}(s) \backslash F$ or $f^{-1}(B(s))=B(s)$ (i.e. the entire basin of attraction of $s$ is connected).

This immediately implies:

Corollary 1.2. Let $E \subset B(s)$ be an open connected set such that $s \in E$ and $\partial E$ consists of a finite number of disjoint Jordan curves. Suppose there exists a component $F$ of $f^{-1}(E)$ containing $\mathrm{cl} E$. If

$$
\left.\operatorname{deg} f\right|_{F}=\left.\operatorname{deg} f\right|_{B(s)}<\operatorname{deg} f,
$$

then $f$ has degree greater than one on some component of $\partial F$.

Using Theorem 1.1, we are able to prove

Theorem 1.3. Let $f: \widehat{\mathbb{C}} \rightarrow \widehat{\mathbb{C}}$ be a rational map of degree $d \geq 3$. Suppose that there exists an attracting fixed point $s$ whose immediate basin of attraction $B(s)$ contains at least all but two (i.e. $2 d-4)$ critical points or values counted with multiplicity. Then $f$ has degree $d$ on $B(s)$ (or, equivalently, the entire basin of attraction of $s$ is connected).

Received by the editors May 1, 1996 and, in revised form, November 14, 1996.

1991 Mathematics Subject Classification. Primary 58F23.

Research supported by Polish KBN Grant No 2 P301 01307. 
On the other hand, if the number of critical points in $B(s)$ is smaller, then the degree of $f$ on $B(s)$ can be less than $d$.

Theorem 1.4. For $d \geq 3$ and $k=1, \ldots, 2 d-5$ there exists a rational map $f$ of degree $d$ having an attracting fixed point $s$ such that $B(s)$ contains exactly $k$ critical points (counted with multiplicity) and the entire basin of attraction of $s$ is not connected.

These examples also show that in Theorem 1.1 the assumption about the degree of $f$ on the components of $\partial F$ cannot be omitted.

This work is connected with the paper [P1] by F. Przytycki. He proved that if $f \in H^{d}$ (where $H^{d}$ denotes the set of all rational maps of degree $d$ which are expanding on the Julia set) and all but one of the critical points or values (counted without multiplicity) are in the immediate basin $B(s)$ of an attracting fixed point $s$, then $\left.\operatorname{deg} f\right|_{B(s)}=d$ and, moreover, the component of $H^{d}$ containing $f$ also contains a polynomial. If all critical points of $f$ are in the immediate basin of an attracting fixed point or the forward-invariant basin of a parabolic fixed point, then the Julia set is a Cantor set and $f$ on the Julia set is conjugate to the full one-sided shift. These facts were also proved by P. Makienko in [Ma].

Note that if for a rational map $f$ the immediate basin of an attracting fixed point $B(s)$ is simply connected, then it contains exactly $\left.\operatorname{deg} f\right|_{B(s)}-1$ critical points (here and in the sequel we count critical points with multiplicity). If $B(s)$ is not simply connected, then it must contain at least two critical points. However, F. Przytycki has constructed examples of so-called exotic basins, i.e. non-simplyconnected immediate basins $B(s)$ such that $\left.\operatorname{deg} f\right|_{B(s)}=\operatorname{deg} f=d \geq 3$ and $B(s)$ contains exactly $k$ critical points with an arbitrary $k=2, \ldots, 2 d-2$. Our work answers a question asked in [P1] and simplifies construction of the exotic basins.

Remark. Note that Theorem 1.3 depends on holomorphic properties. One can construct a smooth branched covering of $S^{2}$ fulfilling the assumptions of Theorem 1.3 such that $B(s)$ is not connected (cf. the Remark after Corollary 2.3). Here we present figures of basins of infinity for a rational map and for a branched covering of $S^{2}$ (Figure 1).
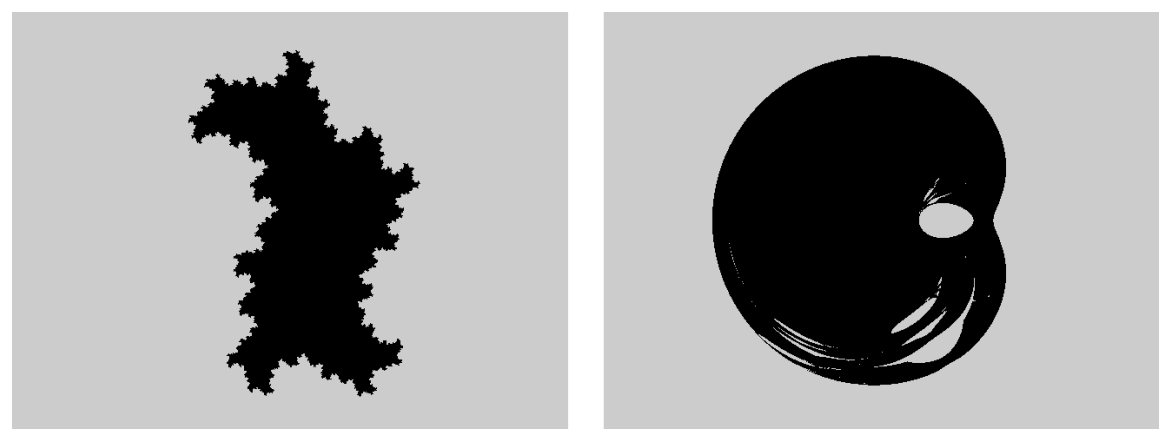

Figure 1. Basin of infinity (gray) for a holomorphic map (left) and for a non-holomorphic branched cover (right).

Example 1.5. There exist rational maps of degree $d$ with disconnected basin of attraction, and $B(s)$ containing all but two critical points counted without multiplicity. To see this, consider the polynomial $p(z)=z^{3}+\frac{3}{2} z$. It has two fixed critical 
points $s_{1}=i \frac{\sqrt{2}}{2}, s_{2}=-i \frac{\sqrt{2}}{2}$. The Julia set of $p$ is connected, so all components of basins of $s_{1}$ and $s_{2}$ are simply connected. Therefore the immediate basin $B\left(s_{1}\right)$ contains one critical point, i.e. all but two critical points of $p$ (as a rational map) counted without multiplicity, but $\left.\operatorname{deg} p\right|_{B\left(s_{1}\right)}=2$. Similar examples can be found for $d>3$.

I would like to express my gratitude to F. Przytycki for fruitful discussions and suggestions which gave rise to many ideas in this paper.

We will often use the following (see e.g. [Mi]):

Riemann-Hurwitz formula. Let $A, B$ be compact Riemann surfaces with smooth boundaries and let $f: A \stackrel{\text { onto }}{\longrightarrow} B$ be a branched covering without branch points on the boundary. Then

$$
\chi(B) \operatorname{deg} f-\chi(A)=\text { Crit },
$$

where Crit is the number of branch points (counted with multiplicity) and $\chi$ is the Euler characteristic. The formula remains true if $f$ is a proper map between non-compact Riemann surfaces.

Notation. Let $U$ be a bounded set in $\mathbb{C}$. A bounded (unbounded) connected component of $\widehat{\mathbb{C}} \backslash U$ will be called an inner (outer) component. Let $\partial U$ denote the boundary of $U$. A connected component of $\partial U$ will be called an inner (outer) component of $\partial U$ if it is a part of the boundary of an inner (outer) component of $\widehat{\mathbb{C}} \backslash U$. If $A$ is a topological annulus, then $\bmod A$ will denote the modulus of $A$.

\section{Proofs of Theorems 1.1 And 1.3}

We introduce here a notion of rational-like mappings. This is a wider class than the so-called generalized polynomial-like maps. Recall that a generalized polynomial-like map is a map from a finite collection of topological discs $U_{i}$ with smooth boundaries onto a topological disc $V$ such that $\operatorname{cl} U_{i} \subset V$ are pairwise disjoint and $\left.f\right|_{U_{i}}$ is a proper holomorphic map onto $V$ of a finite degree. Such a map is quasiconformally conjugate to a polynomial, as was proved in e.g. [LM]. In the definition of rational-like maps we allow $U_{i}$ to have a finite number of holes, i.e. they are finitely connected sets. Moreover, we replace one disc $V$ by a finite number of disjoint topological discs $V_{j}$. We give the precise definition.

Definition 2.1. Let $\left\{U_{i}\right\}_{i=1}^{k}$ be a collection of open domains in $\mathbb{C}$ such that $\operatorname{cl} U_{i}$ are pairwise disjoint and, for every $i, \partial U_{i}$ consists of a finite number of disjoint smooth Jordan curves. Let $\left\{V_{j}\right\}_{j=1}^{n}$ be a collection of bounded topological discs in $\mathbb{C}$ with smooth boundaries such that $\mathrm{cl} V_{j}$ are pairwise disjoint. Assume that

$$
\bigcup_{i=1}^{k} \operatorname{cl} U_{i} \subset \bigcup_{j=1}^{n} V_{j} .
$$

Let $f: \bigcup_{i=1}^{k} U_{i} \stackrel{\text { onto }}{\longrightarrow} \bigcup_{j=1}^{n} V_{j}$ be a holomorphic map extendable to the boundary of $\bigcup_{i} U_{i}$. Assume that for every $i$ there exists $j(i)$ such that $f\left(U_{i}\right)=V_{j(i)}$ and for every component $\delta$ of $\left.\partial U_{i} f\right|_{\delta}: \delta \stackrel{\text { onto }}{\longrightarrow} \partial V_{j(i)}$ is a covering of finite degree. Then we will call $\left(\left\{U_{i}\right\}_{i},\left\{V_{j}\right\}_{j}, f\right)$ a rational-like mapping.

As for polynomial-like mappings, define the filled-in Julia set $K(f)$ to be the set of points for which the entire forward trajectory is contained in $\bigcup_{i=1}^{k} U_{i}$. 
A question arises, whether a rational-like map is quasiconformally conjugate to a rational map such that $\widehat{\mathbb{C}} \backslash \bigcup_{i} U_{i}$ is contained in the immediate basin of attraction of an attracting fixed point. It is not difficult to show that this holds when $n=1$, i.e. all sets $U_{i}$ are mapped onto the same topological disc $V$ (Lemma 3.1 in Section 3). If $n>1$, then obviously one must add some combinatorial assumptions about the degree of $f$ on the boundaries of $U_{i}$. For instance, one can prove that in the case when all $U_{i}$ are simply connected and

$$
\left.\operatorname{deg} f\right|_{f^{-1}\left(V_{m}\right) \cap V_{j}}=d_{j}
$$

for all $j, m=1, \ldots, n$, then $f$ is quasiconformally conjugate to a polynomial of degree $d=d_{1}+\ldots+d_{n}$. In the general case one must add some more combinatorial assumptions. For details, see [Ba].

The following lemma will be the main tool to prove Theorem 1.1.

Lemma 2.2. Let $f: \bigcup_{i=1}^{k} U_{i} \stackrel{\text { onto }}{\longrightarrow} \bigcup_{j=1}^{n} V_{j}$ be a rational-like map such that at least one of the sets $U_{i}$ is not simply connected and for every $i=1, \ldots, k$ we have $\operatorname{deg} f=1$ on the outer component of $\partial U_{i}$. Then the unbounded component of $\widehat{\mathbb{C}} \backslash K(f)$ contains at least one bounded component of $\widehat{\mathbb{C}} \backslash \bigcup_{i=1}^{k} U_{i}$.

Proof. Let $D_{i}^{(1)}$ be a topological disc which is the union of $U_{i}$ and all inner components of $\widehat{\mathbb{C}} \backslash U_{i}$. Renumbering $U_{i}$, we can assume that for some $m_{1} \leq k$ we have $\bigcup_{i=1}^{k} D_{i}^{(1)}=\bigcup_{i=1}^{m_{1}} D_{i}^{(1)}$, and $\operatorname{cl} D_{1}^{(1)}, \ldots, \operatorname{cl} D_{m_{1}}^{(1)}$ are pairwise disjoint. (Note that $m_{1}$ can be less than $k$, since some of the sets $U_{i}$ can be contained in inner components of $\widehat{\mathbb{C}} \backslash U_{j}$ for some $j \neq i$.) We will call $D_{1}^{(1)}, \ldots, D_{m_{1}}^{(1)}$ discs of the 1 st generation. By definition, $K(f) \subset \bigcup_{i=1}^{m_{1}} D_{i}^{(1)}$.

Let

$$
a=\min _{j=1, \ldots, n} \bmod A_{j}
$$

for some topological annuli $A_{j}$ such that the outer component of $\partial A_{j}$ is equal to $\partial V_{j}$ and the inner component of $\mathbb{C} \backslash A_{j}$ contains $\bigcup_{i=1}^{m_{1}} D_{i}^{(1)} \cap V_{j}$. By the Grötzsch inequality, for every $i, j$ such that $D_{i}^{(1)} \subset V_{j}$ we have

$$
\bmod \left(V_{j} \backslash D_{i}^{(1)}\right) \geq a \text {. }
$$

Consider $f^{-1}\left(\bigcup_{i=1}^{m_{1}} D_{i}^{(1)}\right)$. Denote its components by $U_{1}^{(2)}, U_{2}^{(2)}, \ldots$ Let $D_{i}^{(2)}$ be a topological disc which is the union of $U_{i}^{(2)}$ and all inner components of $\widehat{\mathbb{C}} \backslash U_{i}^{(2)}$. As previously, for some $m_{2}$ we can assume $\bigcup_{i} D_{i}^{(2)}=\bigcup_{i=1}^{m_{2}} D_{i}^{(2)}$ and $\operatorname{cl} D_{1}^{(2)}, \ldots, \operatorname{cl} D_{m_{2}}^{(2)}$ are pairwise disjoint. Moreover, $K(f) \subset \bigcup_{i=1}^{m_{2}} D_{i}^{(2)} \subset \bigcup_{i=1}^{m_{1}} D_{i}^{(1)}$. We will called $D_{1}^{(2)}, \ldots, D_{m_{2}}^{(2)}$ discs of the 2 nd generation. In the same way we define $\operatorname{discs} D_{1}^{(n)}, \ldots, D_{m_{n}}^{(n)}$ of the $n$th generation. To simplify notation, define $D_{j}^{(0)}=V_{j}$ to be discs of the zero generation.

By construction, for all $n$

$$
K(f) \subset \bigcup_{i=1}^{m_{n+1}} D_{i}^{(n+1)} \subset \mathrm{cl} \bigcup_{i=1}^{m_{n+1}} D_{i}^{(n+1)} \subset \bigcup_{i=1}^{m_{n}} D_{i}^{(n)}
$$

and $\operatorname{cl} D_{1}^{(n)}, \ldots, \operatorname{cl} D_{m_{n}}^{(n)}$ are pairwise disjoint. We will show that there exists $n_{0}$ such that $\widehat{\mathbb{C}} \backslash \bigcup_{i=1}^{m_{n_{0}}} D_{i}^{\left(n_{0}\right)}$ contains at least one inner component of the boundary of some $U_{i}$. (This will end the proof.) 
Note that $\widehat{\mathbb{C}} \backslash D_{i}^{(n)}$ can contain a critical point of $f$ from $K(f)$ for only a finite number of $n$. (Otherwise there would exist a critical point $c$ such that $c \in K(f)$ and $f^{n}(c) \notin \bigcup_{i} U_{i}$ for some $n$, which is impossible.) Therefore, if we enlarge the $\operatorname{discs} D_{i}^{(1)}$ slightly, we can assume that for all $n \geq 1$ and for all $i$ there are no critical points in $\partial D_{i}^{(n)}$.

Let $D_{i}^{(1)}$ be a disc of the 1 st generation and let $j$ be such that $f\left(\partial D_{i}^{(1)}\right)=\partial D_{j}^{(0)}$. Let $D_{i_{1}}^{(1)}, \ldots, D_{i_{l}}^{(1)}$ be all discs of the 1 st generation contained in $D_{j}^{(0)}$. Denote by $W$ the component of $f^{-1}\left(D_{j}^{(0)} \backslash \operatorname{cl} \bigcup_{s=1}^{l} D_{i_{s}}^{(1)}\right)$ for which $\partial W \supset \partial D_{i}^{(1)}$. Suppose that $\left.\operatorname{deg} f\right|_{W}>1$. By assumption, $\left.\operatorname{deg} f\right|_{\partial D_{i}^{(1)}}=\left.\operatorname{deg} f\right|_{\text {outer comp. of } \partial U_{i}}=1$, so $f^{-1}\left(\partial D_{j}^{(0)}\right) \cap \partial W$ has at least one component other than $\partial D_{i}^{(1)}$. This is an inner component of $\partial U_{i}$, and it is easy to see that it lies outside all discs of the 2nd generation, so the proof ends in this case. Therefore we can assume that $\left.\operatorname{deg} f\right|_{W}=$ 1 for all $i \leq m_{1}$. Since there are no critical points in $\partial W,\left.\operatorname{deg} f\right|_{\partial W}=1$; so $f$ has degree 1 on the boundary of all discs of the second generation contained in $D_{i}^{(1)}$. Moreover, $W$ contains the biholomorphic preimage of the annulus $A_{j}$ separating $\partial D_{i}^{(1)}$ from all discs of the second generation contained in $D_{i}^{(1)}$. Since this holds for all $i \leq m_{1}$, it follows that $f$ has degree 1 on the boundary of all discs of the second generation, and

$$
\bmod \left(V_{j} \backslash D_{i}^{(2)}\right) \geq 2 a
$$

for every $j$ and every $D_{i}^{(2)} \subset V_{j}$.

Now we can replace $D_{i}^{(1)}$ by $D_{i}^{(2)}, D_{j}^{(0)}$ by $D_{j}^{(1)}$ and repeat the arguments from the previous paragraph. If $\left.\operatorname{deg} f\right|_{W}>1$ for some $i$, then we stop; otherwise we can repeat the construction inductively. Note that if the construction does not end after $n$ steps, then we have

$$
\bmod \left(V_{j} \backslash D_{i}^{(n)}\right) \geq n a .
$$

for every $j$ and and every $D_{i}^{(n)} \subset V_{j}$. By assumption, there exists an inner component $C$ of the boundary of some $U_{i}$, and the construction shows that $C \subset \bigcup_{i} D_{i}^{(n)}$. But on the other hand (1) implies that diameters of all $D_{i}^{(n)}$ are arbitrarily small when $n$ is sufficiently large. Hence the construction must stop after a finite number of steps, which means that there exist $n_{0}, i, j$ such that $f\left(\partial D_{i}^{\left(n_{0}\right)}\right)=\partial D_{j}^{\left(n_{0}-1\right)}$, $D_{i_{1}}^{\left(n_{0}\right)}, \ldots, D_{i_{l}}^{\left(n_{0}\right)}$ are all discs of the $n_{0}$ th generation contained in $D_{j}^{\left(n_{0}-1\right)}, W$ is the component of $f^{-1}\left(D_{j}^{\left(n_{0}-1\right)} \backslash \operatorname{cl} \bigcup_{s=1}^{l} D_{i_{s}}^{\left(n_{0}\right)}\right)$ for which $\partial W \supset \partial D_{i}^{\left(n_{0}\right)}$, and $\left.\operatorname{deg} f\right|_{\mathrm{cl} W}>1$. Hence there exists a component $\delta$ of $f^{-1}\left(\partial D_{j}^{\left(n_{0}-1\right)}\right) \cap \partial W$ other than $\partial D_{i}^{\left(n_{0}\right)}$. Note that $\delta$ does not intersect discs of the $\left(n_{0}+1\right)$ th generation. Let $p$ be such that $D_{j}^{\left(n_{0}-1\right)}$ is contained in $V_{p}$. Connect $\partial D_{j}^{\left(n_{0}-1\right)}$ to $\partial V_{p}$ by a simple curve $\gamma$ lying outside discs of the $\left(n_{0}-1\right)$ th generation. Consider a component $\gamma^{\prime}$ of $f^{-1}(\gamma)$ which begins at some point of $\delta$. Note that $\gamma^{\prime}$ does not intersect the boundary of any disc of the $\left(n_{0}+1\right)$ th generation, since $\gamma$ is disjoint from the boundaries of discs of the $n_{0}$ th generation. Moreover, $\gamma^{\prime}$ does not intersect $W$ because $\gamma$ lies outside $D_{j}^{\left(n_{0}-1\right)}$. Hence $\gamma^{\prime}$ lies in one of inner components of $\widehat{\mathbb{C}} \backslash W$, so it ends at an inner component $C$ of the boundary of some $U_{i}$. Suppose that $C$ intersects a disc $D$ of the $\left(n_{0}+1\right)$ th generation. Since $C$ cannot intersect $\partial D$, it follows that 
$C \subset D$. But this is impossible, since we have shown that $\gamma^{\prime} \cap \partial D=\emptyset=\delta \cap D$. Therefore $C$ is disjoint from discs of the $\left(n_{0}+1\right)$ th generation. This finishes the proof.

Consider a particular case of Lemma 2.2, when $k=n=1$. This means that $f$ is a map from a finitely connected set $U$ onto a topological disc $V$. Then we can state the following corollary (we will not use it in the remaining part of the paper).

Corollary 2.3. Let $U \subset \mathbb{C}$ be an open domain with boundary consisting of finitely many (at least two) disjoint Jordan curves, let $V$ be a bounded topological disc in $\mathbb{C}$ such that $\mathrm{cl} U \subset V$, and let $f: U \stackrel{\text { onto }}{\longrightarrow} V$ be a proper holomorphic map of finite degree. Suppose that $\operatorname{deg} f=1$ on the outer component of $\partial U$. Then there exist a critical point $c$ of $f$ and a number $n \geq 1$ such that $f^{n}(c) \in V \backslash \operatorname{cl} U$.

Proof. Suppose that forward trajectories of all critical points are contained in $\operatorname{cl} U$. Repeat the proof of Lemma 2.2. We claim that at each step of the construction we have only one topological disc $D^{(n)}$ of the $n$th generation such that $D^{(n)} \supset D^{(n+1)}$ and $\left.\operatorname{deg} f\right|_{\partial D^{(n)}}=1$. To prove this inductively, suppose that $D^{(n)} \subset D^{(n-1)}$. By assumption, there are no critical values in the topological annulus $A=D^{(n-1)} \backslash D^{(n)}$ so by the Riemann-Hurwitz formula the component $W$ of $f^{-1}(A)$ is also an annulus. By induction, $\left.\operatorname{deg} f\right|_{\partial D^{(n)}}=1$, so $\left.\operatorname{deg} f\right|_{A}=1$, and we have only one disc of the $(n+1)$ th generation, $D^{(n+1)} \subset D^{(n)}$, such that $\left.\operatorname{deg} f\right|_{\partial D^{(n+1)}}=1$. Moreover, for each $n$

$$
\bmod \left(D^{(n)} \backslash D^{(n+1)}\right)=\bmod \left(V \backslash D^{(1)}\right),
$$

so $\bigcap_{n>0} D^{(n)}$ is one point. This is a contradiction, because $\bigcap_{n>0} D^{(n)}$ contains all inner components of $\partial U$.

Remark. The above lemma depends on holomorphic properties. Indeed, consider an open unit $\operatorname{disc} V$, an annulus $U$ such that $\operatorname{cl} U \subset V$, and a holomorphic map $f: U \stackrel{\text { onto }}{\longrightarrow} V$ of degree two. Denote the critical points of $f$ by $c_{1}, c_{2}$. Taking suitable $U$ and $f$, we can assume $c_{1}=f\left(c_{1}\right)=0$ and $\operatorname{Arg} f\left(c_{2}\right)=\operatorname{Arg} c_{2}$. Take a homeomorphism $h(z)=|z|^{\alpha} z$ for $\alpha>0$. Then $\tilde{f}=h \circ f$ is a smooth branch covering of degree two from $U$ onto $V$, and $c_{1}, c_{2}$ are $\tilde{f}$-critical points. For a suitable $\alpha$ we have $\tilde{f}\left(c_{1}\right)=c_{1}$ and $\tilde{f}\left(c_{2}\right)=c_{2}$, so the above corollary will be false. (See Figure 1.)

Proof of Theorem 1.1. By a conformal change of coordinates assume that $s=\infty$. By assumption, $\partial E$ does not contain critical values of $f$, and we can assume that it is smooth. Denote components of $\widehat{\mathbb{C}} \backslash \mathrm{cl} E$ by $V_{1}, \ldots, V_{n}$. Since $E$ is connected, $V_{j}$ are topological discs in $\mathbb{C}$. Let $U_{1}, \ldots, U_{k}$ be all components of $f^{-1}\left(\bigcup_{j=1}^{n} V_{j}\right)$ whose boundaries intersect $\partial F$. If all $U_{i}$ are simply connected then $F \supset f^{-1}(\infty)$, so $f^{-1}(B(\infty))=B(\infty)$, and the proof is finished. Otherwise $\left.f\right|_{\bigcup_{i=1}^{k} U_{i}}$ is a rational-like map satisfying the assumptions of Lemma 2.2. Therefore the outer component of $\widehat{\mathbb{C}} \backslash K\left(\left.f\right|_{\cup_{i} U_{i}}\right)$ contains an inner component $C$ of $\partial U_{i}$ for some $i$. This implies that $C \subset B(\infty)$. Since $C$ is the outer component of the boundary of some component of $f^{-1}(E)$, it follows that $B(\infty)$ contains some point of $f^{-1}(\infty)$ which lies in $U_{i}$. This ends the proof.

Proof of Theorem 1.3. Let $f^{\prime}(s)=\lambda$. Assume $\lambda \neq 0$. Let $\Phi$ be the map which conjugates $f$ to $z \mapsto \lambda z$ in the neighbourhood of $s$, i.e. $\Phi(f(z))=\lambda \Phi(z)$. (If $\lambda=0$ 
then instead of $z \mapsto \lambda z$ we take $z \mapsto z^{k}$ for the suitable $k$ and proceed in the similar way.) Extend $\Phi$ to $B(s)$, putting $\Phi(z)=\lambda^{-n} \Phi\left(f^{n}(z)\right)$. Set $G(z)=|\Phi(z)|$ for $z \in B(s)$. By definition, we have

$$
G(f(z))=|\lambda| G(z)<G(z) .
$$

Connect each $f$-critical value $f\left(c_{i}\right)$ in $B(s)$ to $s$ by a curve $\alpha_{i}$ contained in $B(s)$. $G$ is continuous on $B(s)$, so we can take a number $M$ greater than $\max _{i} \max _{z \in \alpha_{i}} G(z)$. Let $E$ be the component of $\{z: G(z)<M\}$ containing $s$. We can choose $M$ so that $\partial E$ does not intersect backward trajectories of critical points and does not contain critical values. Then $\partial E$ is composed of a finite number of pairwise disjoint smooth Jordan curves, and $\widehat{\mathbb{C}} \backslash \operatorname{cl} E=\bigcup_{j=1}^{n} V_{j}$ for disjoint topological discs $V_{j}$. Let $F$ be the component of $f^{-1}(E)$ containing $E$. As previously, denote by $U_{1}, \ldots, U_{k}$ all components of $f^{-1}\left(\bigcup_{j=1}^{n} V_{j}\right)$ whose boundaries intersect $\partial F$. By assumption, there are at most two critical points (counted with multiplicity) in $\bigcup_{i=1}^{k} U_{i}$. If all $U_{i}$ are simply connected, then $\bigcup_{i=1}^{k} U_{i}=\widehat{\mathbb{C}} \backslash \operatorname{cl} F$ and $f^{-1}(\partial E)=\partial F$, so $f^{-1}(B(s))=B(s)$. Suppose that, for some $i_{0}, U_{i_{0}}$ is not simply connected. From the Riemann-Hurwitz formula we conclude that $\chi\left(U_{i_{0}}\right) \geq\left.\operatorname{deg} f\right|_{U_{i_{0}}}-2$. This implies that $U_{i_{0}}$ is a topological annulus, $U_{i_{0}}$ contains two single critical points and $\left.\operatorname{deg} f\right|_{U_{i_{0}}}=2$. Hence $f$ has degree 1 on each component of $\partial U_{i_{0}}$. Moreover, for every $i \neq i_{0}, U_{i}$ does not contain critical points; so it is a topological disc and $\left.\operatorname{deg} f\right|_{\partial U_{i}}=1$. By Theorem 1.1, $B(s)$ contains a point from $f^{-1}(s) \backslash F$. Denote by $W_{1}, \ldots, W_{m}$ all components of $f^{-1}\left(\bigcup_{j=1}^{n} V_{j}\right) \backslash \bigcup_{i=1}^{k} U_{i}$. Since there are no critical points in $\bigcup_{i=1}^{m} W_{i}$, every $W_{i}$ is a topological disc in $\widehat{\mathbb{C}} \backslash\left(\operatorname{cl} F \cup \operatorname{cl} U_{i_{0}}\right)$. Hence $f^{-1}(E)$ has exactly two components: $F$ and $\tilde{F}=\widehat{\mathbb{C}} \backslash\left(\operatorname{cl} F \cup \operatorname{cl} U_{i_{0}} \cup \operatorname{cl} \bigcup_{i=1}^{m} W_{i}\right)$. It follows that $B(s)$ contains a point from $f^{-1}(s) \cap \tilde{F}$; so $f^{-1}(s) \subset B(s)$. The theorem is proved.

\section{RATIONAL-LIKE MAPS AND ThEOREM 1.4}

The examples in Theorem 1.4 will be constructed from some rational-like maps by the use of the quasiconformal surgeries technique (see $[\mathrm{DH}]$ ). First we show that if all sets $U_{i}$ in Definition 2.1 are mapped onto one topological disc $V$, then the rational-like map $f$ is quasiconformally conjugate to a rational map.

Lemma 3.1. Let $f: \bigcup_{i=1}^{k} U_{i} \rightarrow V$ be a rational-like map onto a topological disc $V$. Let $d=\left.\sum_{i=1}^{k} \operatorname{deg} f\right|_{\partial U_{i}}$. Then there exist a rational map $R$ of degree $d$ and a quasiconformal homeomorphism $h$ of $\widehat{\mathbb{C}}$ conjugating $f$ with $\left.R\right|_{h\left(\cup_{i} U_{i}\right)}$. If each $U_{i}$ is a topological disc, then $R$ is conformally conjugate to a polynomial.

In the proof we will use the following combinatorial lemma:

Lemma 3.2. Let $a_{1}, \ldots, a_{k}$ be disjoint points in $\widehat{\mathbb{C}}$, and let $d_{1}, \ldots, d_{k}$ be positive integers. Let $D$ be an open topological disc in $\widehat{\mathbb{C}}$ with smooth boundary and let $a \in \widehat{\mathbb{C}} \backslash \mathrm{cl} D$. Then there exist disjoint open topological discs $D_{1}, \ldots, D_{k}$ with smooth boundaries and a rational map $g$ of degree $\sum_{i=1}^{k} d_{i}$ such that:

1. $g^{-1}(\operatorname{cl} D)=\widehat{\mathbb{C}} \backslash \bigcup_{i=1}^{k} D_{i}, a_{i} \in D_{i}$ and $g\left(a_{i}\right)=a$.

2. $\left.g\right|_{\partial D_{i}}: \partial D_{i} \stackrel{\text { onto }}{\longrightarrow} \partial D$ is a covering of degree $d_{i}$.

If $a_{i} \in \mathbb{C}$ for all $i$ and $\infty \in D$, then we can choose $g$ to be a polynomial. 
Proof. Assume first that $a_{i} \in \mathbb{C}$ for all $i$ and $\infty \in D$. Let $h$ be an affine map such that $h(\widehat{\mathbb{C}} \backslash \mathrm{cl} D)$ is a small topological disc around 0 and $h(a)=0$. Take

$$
p(z)=\prod_{i=1}^{k}\left(z-a_{i}\right)^{d_{i}}
$$

and let $D_{i}$ be the component of $p^{-1}(h(\widehat{\mathbb{C}} \backslash \operatorname{cl} D))$ containing $a_{i}$. Then $g=h^{-1} \circ p$ is the required polynomial. For the general case it is sufficient to take $h_{1} \circ g \circ h_{2}$, where $h_{1}, h_{2}$ are suitable homographies.

Proof of Lemma 3.1. We will extend $f$ to a smooth branch covering $F$ of $\widehat{\mathbb{C}}$ which preserves a bounded conformal structure $\mu$. Define $D=\widehat{\mathbb{C}} \backslash \operatorname{cl} V$. Let $W$ be an inner component of $\widehat{\mathbb{C}} \backslash \operatorname{cl} \bigcup_{i} U_{i}$. We want to have

$$
F: \operatorname{cl} W \stackrel{\text { onto }}{\longrightarrow} \mathrm{cl} D \text {. }
$$

To construct such an $F$ on $\mathrm{cl} W$ which is consistent with $f$ on $\partial W$, it is sufficient to take a suitable rational map from Lemma 3.2 composed with a smooth homeomorphism of $\widehat{\mathbb{C}}$. Let $W_{0}$ be the component of $V \backslash \bigcup_{i} U_{i}$ such that the outer component of $\partial W_{0}$ is equal to $\partial V$. Define $F$ on $\operatorname{cl} D$ so that $F(\infty)=\infty, \operatorname{cl} F(D) \subset D$ and $F$ on $\operatorname{cl} D$ is conformally conjugate to $z^{d^{\prime}}$, where $d^{\prime}$ is the sum of the degrees of $f$ on inner components of $\partial W_{0}$. Let $A=D \backslash \operatorname{cl} F(D)$. Modifying Lemma 3.2, we can easily define $F$ to map $\operatorname{cl} W_{0}$ onto $\mathrm{cl} A$ so that $F$ on $\operatorname{cl} W_{0}$ is a smooth branch covering consistent with the previously defined $F$ on $\partial W_{0}$. By construction, $F: \widehat{\mathbb{C}} \rightarrow \widehat{\mathbb{C}}$ is a smooth branch covering of degree $d$ which is conformal on $D \cup \bigcup_{i} U_{i}$.

Let $\mu_{0}$ be the standard conformal structure on $\widehat{\mathbb{C}}$. Define a conformal structure $\mu$ :

$$
\mu= \begin{cases}\mu_{0} & \text { on } D, \\ \left(F^{n}\right)^{*}\left(\left.\mu\right|_{D}\right) & \text { on } F^{-n}(D), \\ \mu_{0} & \text { else. }\end{cases}
$$

Then $F$ preserves $\mu$ a.e. on $\widehat{\mathbb{C}}$. Moreover, $\mu \in L^{\infty}$, because for a.e. point $z \in \widehat{\mathbb{C}}$ in the forward trajectory of $z$ there is at most one point where $F$ is not conformal. Using the measurable Riemann theorem, we can integrate $\mu$, and after a quasiconformal change of coordinates $F$ preserves the standard structure a.e. on $\widehat{\mathbb{C}}$, so it is a rational map of degree $d$. By construction, if all $U_{i}$ are simply connected, then this rational map will be conformally conjugate to a polynomial. (This is the case for generalized polynomial-like maps.)

In the proof of Theorem 1.4 we will use some properties of Newton maps for polynomials. Recall that the Newton map for a polynomial $p$ of degree $d$ with simple roots is the rational map given by

$$
N(z)=z-\frac{p(z)}{p^{\prime}(z)} .
$$

Then $N$ has $d$ superattracting fixed points, namely the roots of $p$, and the remaining $N$-critical points are the roots of $p^{\prime \prime}$.

Moreover, for a Newton map for any polynomial the components of the basins of the supersinks are topological discs (see [P2]) and the Julia set is connected (see 
[Sh]). Recall also that any rational map of degree $d$ having $d$ superattracting fixed points is holomorphically conjugate to a Newton map for some polynomial of degree $d$. For more detailed information on Newton maps, refer to e.g. [HP].

Proof of Theorem 1.4. For $k=1$ it is sufficient to take a Newton map of the suitable degree. This also covers the case $d=3$. Let $d \geq 4, k \geq 2$. Take a Newton map $N$ of degree three with three supersinks $0,1, \infty$ such that the fourth critical point is not in the basin of $\infty$. Let $\gamma=\{|z|=r\}$ for large $r$. Then $N^{-1}(\gamma)$ consists of two Jordan curves. Let $A$ be the topological annulus for which $\partial A=N^{-1}(\gamma)$. Note that the Julia set of $N$ is contained in $A$, the outer component of $\widehat{\mathbb{C}} \backslash A$ is in the immediate basin of $\infty$, and the inner component of $\widehat{\mathbb{C}} \backslash A$ is in the non-immediate component of the basin of $\infty$. Let $g=N$ on $\operatorname{cl} A$. We have $\operatorname{cl} A \subset g(A)=\{|z|<$ $r\} ; g$ has degree two on the outer part of $\partial A$ and degree one on the inner part of $\partial A$.

Let $1 \leq m \leq d-3$. Take $m$ discs $D_{1}, \ldots, D_{m}$ in the outer component of $\mathbb{C} \backslash \operatorname{cl} A$ such that $\operatorname{cl} D_{i} \subset g(A)$ are pairwise disjoint, and define $g$ on them so that each $D_{i}$ is mapped biholomorphically onto $g(A)$. If $m<d-3$, let $D_{m+1}$ be a disc in the inner component of $\mathbb{C} \backslash \mathrm{cl} A$ and set $g$ to map it onto $g(A)$ with degree $d-m-3$.

Now $g$ on $\bigcup_{i} D_{i} \cup A$ is a rational-like map of degree $d$. By Lemma 3.1 there exist a rational map $f$ of degree $d$ and a quasiconformal homeomorphism $h$ such that $g$ on $\bigcup_{i} D_{i} \cup A$ is conjugate by $h$ to $f$ on $h\left(\bigcup_{i} D_{i} \cup A\right)$. Let $C$ be the outer component of $\widehat{\mathbb{C}} \backslash\left(\bigcup_{i} \operatorname{cl} D_{i} \cup \operatorname{cl} A\right)$. By construction, $h(C)$ is contained in the immediate basin of an attracting fixed point $s$, and by the Riemann-Hurwitz formula there are exactly $2 m+1 f$-critical points in $h(C)$ (counted with multiplicity). By construction, none of the three $f$-critical points in $h(A)$ escape to $s$. All other $f$-critical points are in $h$ (inner component of $\mathbb{C} \backslash A$ ), and they are in a non-immediate component of the basin of $s$ since they are separated from $B(s)$ by the Julia set. This shows that the entire basin of attraction of $s$ is not connected, and $B(s)$ contains exactly $2 m+1$ critical points.

Since $m$ was an arbitrary number between 1 and $d-3$, we have proved the assertion for $k=3,5, \ldots, 2 d-5$. To obtain examples for even $k$ it is sufficient to modify the above construction by taking $f$ on $D_{1}$ to be a map of degree two such that the critical point is fixed and $f$ on $D_{m+1}$ to be a map of degree $d-m-4$. Then the number of critical points in $B(s)$ is $2 m+2$ and $m=1, \ldots, d-4$, so we get $k=4,6, \ldots, 2 d-6$. We are left with $k=2$. To prove this case, take a Newton map $N$ for a polynomial $p$ of degree four with single roots such that for a point $a$ we have $p(a)=p^{\prime \prime}(a)=0$. Then $a$ is a double critical fixed point for $N$, and there are three other supersinks. We can choose $N$ so that the sixth $N$-critical point is outside $B(a)$. Changing the coordinates, assume $a=\infty$. Take $\gamma=\{|z|=r\}$ for large $r$. Then $N^{-1}(\gamma)$ consists of two Jordan curves. As previously, define $A$ to be the topological annulus for which $\partial A=N^{-1}(\gamma)$. The Julia set of $N$ is contained in $A$, the outer component of $\widehat{\mathbb{C}} \backslash A$ is in the immediate basin of $\infty$, and the inner component of $\widehat{\mathbb{C}} \backslash A$ is in the non-immediate component of the basin of $\infty$. Let $g=N$ on $\operatorname{cl} A$. Now $g$ has degree three on the outer part of $\partial A$ and degree one on the inner part of $\partial A$. Let $D$ be a disc in the inner component of $\mathbb{C} \backslash A$. Set $g$ to map $D$ onto $N(A)$ with degree $d-4$. As previously, take a rational map $f$ conjugate to $g$. Arguing as before, we show that $f$ has exactly one double critical point (i.e. two critical points when counted with multiplicity) in the immediate basin of attraction $B(s)$, and the entire basin of $s$ is not connected. 


\section{REFERENCES}

[Ba] K. Barański, Ph.D. thesis, in preparation.

[DH] A. Douady and J. Hubbard, On the dynamics of polynomial-like mappings, Ann. Sci. École Norm. Sup. 18 (1985), 287-343. MR 87f:58083

[HP] F. von Haeseler and H.-O. Peitgen, Newton's method and complex dynamical systems, Acta Appl. Math. 13 (1988), 3-58. MR 90:58102

[LM] M. Lyubich and J. Milnor, The Fibonacci unimodal maps, J. Amer. Math. Soc. 6 (1993), 425-457. MR 93h:58080

[Ma] P. Makienko, Pinching and plumbing deformations of quadratic rational maps, preprint, Internat. Centre Theoret. Phys., Miramare-Trieste, 1993

[Mi] J. Milnor, Dynamics in one complex variable: introductory lectures, preprint, SUNY at Stony Brook, IMS \# 1990/5.

[P1] F. Przytycki, Iterations of rational functions: which hyperbolic components contain polynomials?, Fund. Math. 149 (1996), 95-118. MR 97e:58199

[P2] - Remarks on simple-connectedness of basins of sinks for iterations of rational maps, in: Banach Center Publ. 23, PWN, 1989, 229-235. MR 92e:58180

[Sh] M. Shishikura, The connectivity of the Julia set of rational maps and fixed points, preprint, Inst. Hautes Études Sci., Bures-sur-Yvette, 1990.

Institute of Mathematics, Warsaw University, Ul. Banacha 2, 02-097 Warsaw, Poland

E-mail address: baranski@mimuw.edu.pl 